\title{
Tumor hypoxia impairs NK cell cytotoxicity through SHP-1-mediated attenuation of STAT3 and ERK signaling pathways
}

\section{Yanmeng Wang}

Xi'an Jiaotong University

\section{Rui Teng}

Xi'an Jiaotong University

Nan Lv

Xi'an Jiaotong University

Ramone A. Williamson

Xi'an Jiaotong University

Lei Lei

Xi'an Jiaotong University

\section{Rong Fan}

Xi'an Jiaotong University

\section{Ping Chen}

Xi'an Jiaotong University

\section{Li Lei}

Xi'an Jiaotong University

\section{Yuchen Zhang}

Xi'an Jiaotong University

\section{Baiyan Wang}

Xi'an Jiaotong University

\section{Yue Peng}

Xi'an Jiaotong University

Jiaqi Fu

Xi'an Jiaotong University

Xuna Liu

Xi'an Jiaotong University

Aili He

Xi'an Jiaotong University

\section{Michael O'Dwyer}

National University of Ireland Galway

Jinsong Hu ( $\sim$ jinsong.hu@xjtu.edu.cn ) 
Xi'an Jiaotong University https://orcid.org/0000-0003-0565-4567

\section{Research article}

Keywords: Tumor hypoxia; NK cell; cytotoxicity; SHP-1; ERK; STAT3

Posted Date: October 15th, 2019

DOI: https://doi.org/10.21203/rs.2.10559/v2

License: (c) (1) This work is licensed under a Creative Commons Attribution 4.0 International License. Read Full License 


\section{Abstract}

Natural killer (NK) cells are innate immune effectors with potent anti-tumor activity. Nonetheless, tumor cells have the ability to create an immunosuppressive microenvironment, thereby escaping from immune surveillance. Although accumulating evidence indicates that microenvironmental hypoxia plays an important role in favoring tumor development and immune evasion, it is still unclear how hypoxia directly impairs NK cell anti-tumor activity. In this study, we confirmed that hypoxic NK cells show significantly lower cytotoxicity against tumor cells. Consistent with this, we also found that the reduction in NK cell cytotoxicity resulting from hypoxia is related to the lower expression of granzyme B, IFN- $y$, degranulation marker CD107a, as well as killer activation receptors including NKp30, NKp46, and NKG2D on NK cells. More importantly, we further demonstrated that a reduction in the phosphorylation levels of ERK and STAT3 secondary to hypoxia are tightly associated with the attenuated NK cell cytotoxicity. Focusing on the mechanism responsible for reducing phosphorylation levels of ERK and STAT3, we revealed that the activation of protein tyrosine phosphatase SHP-1 (src homology region 2 domain-containing phosphatase-1) following hypoxia may play an essential role in this process. When knocking down SHP-1 or blocking its activity using a specific inhibitor TPI-1, we were able to partially restore NK cell cytotoxicity under hypoxia. Taken together, we demonstrated that hypoxia can impair NK cell cytotoxicity by decreasing the phosphorylation levels of ERK and STAT3 in a SHP-1 dependent manner. Therefore, targeting SHP-1 could provide an approach to enhance NK cell-based tumor immunotherapy.

\section{Introduction}

Natural killer (NK) cells are cytotoxic innate lymphoid cells involved in the immune surveillance of tumors [1]. NK cells have a natural ability to kill tumor cells without any priming or prior activation, which is distinct from that of adaptive immune cells. Once the decision is made to kill, NK cells will release cytotoxic granules containing perforin and granzyme to lyse the target cells. Activated NK cells can also upregulate death-inducing ligands such as Fas-L and TRAIL which induce apoptosis in target cells via activation of the death receptor pathway [2]. In addition, NK cells can produce a variety of proinflammatory cytokines and chemokines to modulate innate and adaptive immune activities against tumors [3]. NK cell cytotoxicity is tightly regulated by a delicate balance between activating and inhibitory receptors [4]. Activating receptors, such as NKG2D, DNAM-1 and NK cytotoxicity receptors NKp30, NKp44 and NKp46, can recognize specific stress-induced ligands expressed on the surface of tumor cells, enhancing NK cell cytotoxicity [5]. The recognition of cognate MHC (major histocompatibility complex) class I molecules by killer cell immunoglobulin-like inhibitory receptors (KIRs), is important in suppressing the cytotoxic activity of NK cells, against normal healthy cells [6, 7]. Loss of MHC I expression by tumor cells removes this inhibition, leading to enhanced NK cytotoxicity. Apart from the KIRs, a variety of other inhibitory receptors have been described, which may impact on NK cytotoxicity. These include NKG2A, PD-1, TIGIT, CD96, Siglec-7 and Siglec-9 [8-11].

Due to the importance of NK cells in the tumor immune surveillance, NK-cell based immunotherapy is emerging as a novel and promising approach to treating tumors. Successful adoptive NK cell-based 
therapies in preclinical and early clinical phases have been demonstrated in several hematological malignancies, leading to complete remission and increased disease-free survival. However, the results in solid tumors are less encouraging [12]. It has been indicated that the failure of immune surveillance may be partially caused by the emergence of tumor escape variants, due to sustained immunological pressure on tumor cells [13]. Alternatively, accumulating evidence suggests that the tumor microenvironment is involved in tumor evasion from NK cell-mediated killing through cellular and non-cellular mechanisms [14]. In this regard, a clear demonstration of how the tumor microenvironment can affect the phenotype and function of NK cells has not yet been fully elucidated.

Hypoxia is a prominent feature of solid tumors and one of the hallmarks of the tumor microenvironment [15]. More recently, it has also been demonstrated that hypoxia is a prevalent feature of the bone marrow microenvironment in acute myeloid leukemia and multiple myeloma, but not the normal bone marrow [1619]. It is well established that tumor hypoxia is an adverse prognostic and predictive factor, and is involved in fostering many aspects of tumor development. Of note, emerging evidence also indicates that tumor hypoxia is a key factor regulating the loss of immune reactivity either by decreasing tumor cell sensitivity to cytotoxic immune effectors or by promoting immunosuppressive mechanisms $[20,21]$.

In this study, focusing on the roles of hypoxia in NK cell-mediated immune surveillance, we investigated the resultant effects of hypoxia on NK cell cytotoxicity machinery and the underlying mechanisms. We demonstrated that hypoxia can directly impair NK cell cytolytic function by decreasing the expression of the killer activation receptors (KARs) on the NK cell surface. More importantly, we revealed that impaired NK cell cytotoxicity secondary to hypoxia was mediated by increased expression of phosphatase SHP-1, which can catalyze dephosphorylation at the tyrosine sites of ERK and STAT3, thereby attenuating NK cell activation signaling. Our findings demonstrate a previously unknown role for hypoxia in dysfunction of NK cell-mediated tumor surveillance, and suggest that SHP-1 may represent a novel target for preserving NK cell function in tumor patients and improving NK cell-based immunotherapy.

\section{Methods}

\section{Antibodies and reagents}

Antibodies for Western blotting against phospho-Stat3 (\#4113), Stat3 (\#12640), Phospho-p44/42 MAPK (ERK1/2) (\#9106), p44/p42 MAPK (ERK1/2) (\#9102), Phospho-SHP-1 (\#8849), Phospho-SHP-2 (\#5431), HIF-1a (\#14179) and $\beta$-actin (\#58169) were bought from Cell Signaling. Peroxidase-conjugated goat antirabbit lgG (\#111-035-003) or goat anti-mouse IgG (\#115-005-003) were bought from Jackson ImmunoResearch. Antibodies for flow cytometry, Alexa Fluor 647-labeled anti-human perforin (\#563576) was purchased from BD Biosciences. FITC-labeled Annexin V (\#640945), PE-labeled anti-human IFN-Y (\#506506), anti-human/mouse granzyme B (\#372207), APC-labeled anti-human NKp46 (\#137607), antihuman NKp30 (\#325209), anti-human NKG2D (\#320808), anti-human CD2 (\#300214), anti-human CD107a (\#12-1079-42) antibodies, were purchased from Biolegend. Sytox® Green Dead Cell Stain was bought from Molecular Probes (\#S34860). STAT3 inhibitor Cryptotanshinone was bought from Selleck 
Chemicals (\#35825-57-1). SHP-1 inhibitor TPI-1 (\#HY-100463), SHP-2 inhibitor SHP-099 (\#HY-100388) and ERK inhibitor U0126 (\#HY-12031) were bought from MedChemExpress.

\section{Cell culture}

The NK cell line KHYG-1 was cultured in RPMI-1640 (BasalMedium, \#L210KJ) supplemented with 10\% fetal bovine serum (FBS) (Biological Industries, \#04-001-1ACS), $10 \mathrm{ng} / \mathrm{mL}$ human IL-2 (PeproTech, \#20002), $2 \mathrm{mM}$ L-glutamine, $100 \mathrm{U} / \mathrm{mL}$ penicillin and $100 \mu \mathrm{g} / \mathrm{mL}$ streptomycin. The NK cell line NK92 was cultured in Dulbecco's modified Eagle's medium (DMEM) (BasalMedium, \#L110KJ) supplemented with $10 \%$ FBS, $10 \%$ horse serum, $10 \mathrm{ng} / \mathrm{mL} \mathrm{IL}-2,2 \mathrm{mM}$ glutamine, $100 \mathrm{U} / \mathrm{mL}$ penicillin and $100 \mu \mathrm{g} / \mathrm{mL}$ streptomycin. The human multiple myeloma cell line MM.1S and leukemia cell line K562 were grown in RPMI-1640 supplemented with $10 \%$ FBS, $2 \mathrm{mM}$ glutamine, $100 \mathrm{U} / \mathrm{mL}$ penicillin and $100 \mu \mathrm{g} / \mathrm{mL}$ streptomycin. All cells were maintained at $37^{\circ} \mathrm{C}$ in humidified atmosphere containing $5 \% \mathrm{CO}_{2}$. Normoxic or hypoxic cell culture conditions were obtained by culturing cells in a sealed incubator flushed with the mixture of $20 \% \mathrm{O}_{2}, 5 \% \mathrm{CO}_{2}$, and $75 \% \mathrm{~N}_{2}$, or the mixture of $1 \% \mathrm{O}_{2}, 5 \% \mathrm{CO}_{2}$, and $94 \% \mathrm{~N}_{2}$, respectively.

\section{Flow cytometry}

The expression of NK cell cytotoxicity effector molecules and KARs was analyzed by flow cytometry. For membrane staining, $5 \times 10^{5}$ cells were collected and washed with staining buffer (PBS containing $0.1 \%$ $\mathrm{NaN}_{3}$ and $0.1 \% \mathrm{BSA}$ ) three times. The cells were then incubated for 30 min on ice, according to the instructions provided with the respective antibodies. After washing 3 times with cell stain buffer, the cells were resuspended in $300 \mu \mathrm{L}$ staining buffer in the presence of Sytox Green or 7-AAD, which were used to gate out dead cells. Acquisition of 10,000 cells per reaction was performed using a CytoFLEX Cytometer (Beckman Coulter Life Sciences). Data were analyzed with Flowjo v7.6.2 (Tree Star). For intracellular staining, $5 \times 10^{5}$ cells were collected and fixed with $1 \mathrm{~mL} 1 \%$ paraformaldehyde in PBS for 15 minutes at room temperature. After washing 3 times with cell stain buffer, the fixed cells were then resuspended in 2 $\mathrm{mL}$ permeabilization buffer ( $0.1 \%$ saponin in cell staining buffer) and incubated for $30 \mathrm{~min}$ at room temperature. The cells were collected again by centrifugation, stained with antibody at an optimal working concentration in permeabilization buffer for $15 \mathrm{~min}$ on ice. After washing three times with permeabilization buffer, the cells were resuspended cells in 300 uL cell staining buffer for final flow cytometric analysis.

\section{CD107a degranulation assay}

Degranulation of cytotoxic contents from NK cells was measured by analysis of the degranulation marker CD107a by flow cytometry. Briefly, NK cells and tumor cells were individually pre-incubated for $14-16 \mathrm{~h}$ at $20 \%$ or $0 \% \mathrm{O}_{2}$ and after that combined in different effector-to-target (E:T) ratios at either $20 \%$ or $0 \% \mathrm{O}_{2}$ for $4 \mathrm{~h}$. APC labeled anti-CD107a was added to the wells within 5-10 minutes after combining NK and tumor cells. As a positive control, PMA (100 ng/mL, Sigma-Aldrich) and ionomycin (1 mg/mL; Sigma-Aldrich) were added to the NK cells during the $4 \mathrm{~h}$ degranulation assay. 


\section{Flow cytometric cytotoxicity assay}

NK cells and tumor cells were individually pre-incubated for $24 \mathrm{~h}$ at $20 \%$ or $1 \% \mathrm{O}_{2}$ first. NK and target cells were then incubated under comparable conditions in different E:T ratios in a 24 well plate. After the $4 \mathrm{~h}$ incubation at $37^{\circ} \mathrm{C}$ and $5 \% \mathrm{CO}_{2}$, samples were labeled with CD2-APC to distinguish effector from target cells. Cell death was detected with Annexin V-FITC and Sytox Green and analyzed on a flow cytometer. A minimum of 10,000 target events were collected per sample and the results were analyzed using Flowjo v7.6.2.

\section{Western blotting}

For western blotting, treated and untreated KHYG-1 and NK92 cells were lysed in buffer containing $50 \mathrm{mM}$ Tris, $150 \mathrm{mM} \mathrm{NaCl}, 1 \%$ Triton X-100, 1\% sodium deoxycholate, $0.1 \%$ SDS and protease inhibitors on ice for $30 \mathrm{~min}$. Lysates were centrifuged at 12,000 rpm for $15 \mathrm{~min}$ and supernatants were collected. Protein concentration was determined by the BCA protein assay kit (HEART Biotech, \#WB003). Equal amounts of protein were loaded and separated on sodium dodecyl sulfate-polyacrylamide gel electrophoresis gel, and transferred onto a PVDF membrane (Millipore, \#IPVH00010). After blocking for $1 \mathrm{~h}$ with $5 \%$ non-fat milk in PBS with $0.1 \%$ Tween-20 at room temperature, the membrane was incubated with primary antibody at $4{ }^{\circ} \mathrm{C}$ overnight. Immunoblots were visualized using HRP-conjugated secondary antibodies and the ECL Western Blot Detection kit (Phygene Life Sciences, \#PH0353).

\section{siRNA-mediated gene silencing in NK cells}

KHYG-1 cells were kept in the above-mentioned condition. Before siRNA transfection cells were washed in pre-warmed Opti-MEM medium (Life Technologies, Carlsbad, CA, USA) and resuspended in the same medium. $10^{6}$ cells were electroporated with $2 \mu \mathrm{g}$ of siRNA in $100 \mu \mathrm{L}$ Opti-MEM medium in $0.2 \mathrm{~cm}$ cuvettes with an electroporator CUY21EDIT (BEX Co. Ltd, Japan). The electroporation program was set as follows: $P p V=200 \mathrm{~V}, \mathrm{Pp}$ on $10 \mathrm{~ms}$, Pp off $10 \mathrm{~ms}, \mathrm{PdV}=25 \mathrm{~V}, \mathrm{Pd}$ on $50 \mathrm{~ms}, \mathrm{Pd}$ off $50 \mathrm{~ms} ; \mathrm{Pd} \mathrm{N}=10$, capacity=940 $\mu \mathrm{F}$, and exponential decay wave type. Following electroporation, cells were resuspended in $2 \mathrm{~mL}$ complete media. 16-24 $\mathrm{h}$ after electroporation, the cells were used for western blotting or killing assay. Transfection efficiency and viability were analyzed by flow cytometry 2-6 $\mathrm{h}$ after electroporation, to quantitatively measure the expression of fluorescein isothiocyanate (FITC)-labeled siRNA and 7-AAD. SHP-1 mRNA was silenced by using a gene-specific siRNA pool from GenePharma (see Supplementary Table 1).

\section{Statistical analysis}

Statistical analyses were performed using the Prism software package 5.0 (GraphPad Software, San Diego, CA, USA). Data are expressed as the mean \pm SEM of at least three independent experiments. Statistical significance was evaluated by two-tailed paired Student's $t$-test. A $p<0.05\left({ }^{\star}\right),<0.01\left({ }^{\star \star}\right)$, or $<$ $\left.0.001{ }^{\star \star \star}\right)$ was considered statistically significant.

\section{Results}




\section{Hypoxic NK cells show decreased cytotoxicity against tumor cells}

We first investigated whether hypoxia impairs NK cell-mediated lysis of tumor cells. To this end, two NK cell lines KHYG-1 and NK92 were cultured in the presence of IL-2 under hypoxic $\left(1 \% \mathrm{O}_{2}\right)$ or normoxic $(20 \%$ $\mathrm{O}_{2}$ ) conditions for $24 \mathrm{~h}$, and subsequently incubated with the tumor cell lines K562 or MM.1S at different $\mathrm{E}: T$ ratios for $4 \mathrm{~h}$ to evaluate the cytotoxicity by flow cytometry. As shown in Fig. $1 \mathrm{a}$ and $\mathrm{b}$, it revealed that the NK cell cytotoxicity was significantly decreased in $1 \%$ compared to $20 \% \mathrm{O}_{2}$ in both tumor cell lines tested. Meanwhile, we observed a marked accumulation of the hypoxia marker HIF-1a in hypoxic NK cells, whereas it was weak in normoxic NK cells monitored by western blotting (Fig. 1c). Moreover, we excluded the possibility that the decreased cytotoxicity in hypoxia was caused by reduced NK cell viability, since we did not observe increased NK cell death by hypoxia (Fig. 1d).

\section{Hypoxia decreases the expression of cytotoxic effectors and activating receptors on NK cells}

To further explore how hypoxia reduces NK cell killing ability, we measured the expression level of cytotoxic effector granzyme B and perforin. As shown in Fig. 2a, hypoxia-treatment led to decreased secretion of both granzyme $B$ and perforin. Additionally, we observed a reduced expression of the cytokine IFN-y in hypoxic NK cells compared to normoxic condition (Fig. 2b). Importantly, CD107a, which is a degranulation marker of natural killer cell activity, was also diminished by hypoxia (Fig. 2c). Given that a range of receptors that can trigger cytolytic programs, as well as cytokine or chemokine secretion tightly regulates NK cell function, we next evaluated the effects of hypoxia on the expression of the main receptors capable of triggering cytolytic activity. Surface expression of the KARs including NKp46, NKp30, and NKG2D was measured by flow cytometry on both normoxic and hypoxic NK cells. As shown in Fig. $2 d$, it confirmed that hypoxia could decrease the expression of KARs on the NK cell surface.

\section{Hypoxia attenuates ERK and STAT3-mediated NK activation}

It is known that intracellular signals activating NK cell cytokine production and cytotoxic activity are propagated primarily through protein phosphorylation of ERK (extracellular signal-regulated kinase) and STAT3 (signal transducer and activator of transcription 3) [22, 23]. Therefore, we further investigated whether hypoxia could affect the activation of ERK and STAT3, and revealed that hypoxia markedly diminished the phosphorylation level at the tyrosine sites of ERK and STAT3 in the two NK cell lines (Fig. $3 a, b)$. To further validate the effects of the phosphorylation of ERK and STAT3 on the expression of KARs and NK cytotoxicity under hypoxic conditions, we used specific small molecule inhibitors U0126 and cryptotanshinone to block ERK and STAT3 signaling, respectively. As shown in Fig. 3c, inhibition of ERK and STAT3 significantly reduced the expression of NKp30 and NKG2D. Importantly, we found that inhibition of ERK or STAT3 results in significantly impaired cytotoxicity against K562 cells (Fig. 3d).

\section{Hypoxia-decreased phosphorylation level of STAT3 and ERK was mediated by the activation of protein tyrosine phosphatase SHP-1 other than SHP-2}


Cell surface receptors harboring intracytoplasmic tyrosine-based activation motifs (ITAMs) or intracytoplasmic tyrosine-based inhibitory motifs (ITIMs) are often phosphorylated by Src family protein tyrosine kinase (PTK), which in turn creates docking sites for the protein tyrosine phosphatase SHP-1 and SHP-2. Recruitment and activation of the SHP-1 and/or SHP-2 have been demonstrated to be a dominant inhibitory mechanism to prevent the induction of the stimulatory signaling cascade $[24,25]$. In this regard, we further investigated whether phosphatase SHP-1 and SHP-2 are involved in the decrease of ERK and STAT3 phosphorylation by hypoxia. As shown in Fig. 4a and b, hypoxia triggers a significant increase of SHP-1 and SHP-2 phosphorylation. When using a specific SHP-1 inhibitor TPI-1, we observed it could reverse the decrease of the phosphorylation of both ERK and STAT3 (Fig. 4c). Moreover, we also observed that pretreatment with p-SHP1 inhibitor TPI-1 significantly could restore the NK cell cytotoxicity under hypoxia (Fig. 4d). However, we did not observe the same effects when using a specific SHP-2 inhibitor SHP099, which had no effect on the phosphorylation levels of ERK and STAT3, as well as NK cell cytotoxicity (Fig. 5).

\section{Knockdown of SHP-1 rescues of NK cell cytotoxicity in hypoxia}

To further validate the role of SHP-1 in regulating NK cell cytotoxicity, we silenced the gene expression of SHP-1 in KHYG-1 cells and found that knockdown of SHP-1 could increase the phosphorylation level of ERK and STAT3 under hypoxia (Fig. 6a). More importantly, we also confirmed that NK cells with SHP-1 silencing showed enhanced cytotoxicity against K562 cells than control cells in hypoxic condition (Fig. $6 b)$.

\section{Discussion}

In the current study, we investigated how hypoxia affects NK cell activity against tumor cells. We first confirmed that hypoxia could directly impair NK cell cytotoxicity against tumor cells, validated by the decreased expression of granzyme B, perforin, CD107a, and KARs. Additionally, we demonstrated that the inhibition of NK cytotoxicity induced by hypoxia is tightly related to a reduction in the phosphorylation level of ERK and STAT3. More importantly, we further found that activation of the tyrosine phosphatase SHP-1 is responsible for the hypoxia-induced decrease in ERK and STAT3 phosphorylation, thereby controlling NK cytotoxicity (Fig. 6c).

NK cell-based immunotherapy has long been considered a promising approach for tumor treatment. However, altered signaling pathways in tumor cells can orchestrate a suppressive tumor microenvironment to facilitate immune escape. It has been well established that tumor cells exploit cellular and non-cellular strategies to avoid recognition and destruction by the immune system. As a hallmark of tumor microenvironment, tumor hypoxia has long been recognized as a key driver of tumor invasion and metastasis, and resistance to therapy. Focusing on the mechanisms of hypoxia-mediated immune escape in tumor, previous studies mainly focused on the changes in tumors, for instance, hypoxia-induced release of immunosuppressive molecules or expression of immune checkpoint molecules. However, little is known of the direct contribution of hypoxia to NK cell function $[26,27,28]$. 
Therefore, we evaluated the direct effect of hypoxia on NK cell function in vitro and demonstrated that hypoxia could impair NK cell cytotoxicity against tumor cells. This finding is consistent with the results from previous studies in multiple myeloma and hepatitis $C$ virus infection, in which hypoxia directly results in decreased NK cell cytotoxicity [29-31]. In line with this finding, we and others likewise demonstrated that the reduced expression of granzyme B and perforin are tightly related to decreased NK cytolytic function.

NK cells use a combination of receptors and signaling pathways to protect the host against tumors [32]. NK cells express a variety of activating and inhibitory receptors to recognize cellular stress ligands as well as $\mathrm{MHC}$ I and the related molecules, which can lead to NK cell response. These activating receptors initiate PTK-dependent signaling through noncovalent associations with transmembrane signaling adaptors that harbor intracytoplasmic ITAMs. On the other hand, KIRs contain ITIMs to inhibit NK-cellmediated cytotoxicity [33]. Many of these receptors can signal through ITAM-dependent and ITAMindependent pathways to maintain a state of proper responsiveness. In this study, we found that hypoxic NK cells express a lower level of KARs, including NKp30, NKp46, and NKG2D. Our findings are in line with the previous work, in which the authors also observed decreased cell surface expression of KARs and CD16 in association with hypoxia-induced impairment of NK cytotoxicity [29, 30]. However, control of NK cell cytotoxicity is not a simple balance between signals from activating and inhibitory receptors. NK cell function is also tightly regulated by various soluble activators such as cytokines and chemokines [32]. Given the importance of interleukins for NK cell growth, survival, development and cytotoxicity, the culture of NK cells with cytokines such as IL-2 and IL-15 is an approach that permits significant expansion of the NK cell subpopulations in vitro and ex vivo $[34,35]$. For mature NK cells, IL-2 and IL-15 activated signaling pathways including PI3K-AKT-mTOR, JAK-STAT, MEK-ERK have been demonstrated to play pivotal roles in NK cell proliferation and activation [34-36]. Indeed, we noticed that the activation of ERK and STAT3 are necessary for the activation of NK cells, especially during the in vitro culture that is dependent on IL-2. When blocking ERK and STAT3 signaling, NK cell cytotoxicity was significantly reduced. Meanwhile, we also found that the expression of KARs on the NK cell surface was also decreased, suggesting an essential role of ERK and STAT3 signaling in regulating NK cytotoxicity. Of note, we also observed that hypoxia could inactivate the phosphorylation of ERK and STAT3, in line with the lower cytotoxicity of hypoxic NK cells.

With respect to hypoxia-mediated inactivation of ERK and STAT3, our findings further support that tyrosine phosphatase SHP-1 is a key player in this process. It is now accepted that protein tyrosine phosphatase (PTP) SHP-1 and SHP-2 are recruited by activating or inhibitory receptors to diminish ITAM associated tyrosine-phosphorylation [24, 25, 37-40]. In this process, inhibition by ITIM-bearing receptors of signaling mediated by ITAM-coupled receptors is proportional to the magnitude of engagement of both types of receptors by their ligands, thus maintaining a dynamic equilibrium between the activating and inhibitory signals. In this regard, the tyrosine phosphorylation status of the key signaling components that act as substrates for both PTK and PTP is therefore important to the propagation of the NK cell effector pathways. Moreover, the SH2 domain-containing protein tyrosine phosphatase SHP-1 and SHP-2 have been implicated in the regulation of a variety of tyrosine kinase-linked receptors, including cytokine 
and growth factor receptors, integrins, as well as ITAM-containing immune receptors [40, 41]. Firstly, several studies have shown that either SHP-1 or SHP-2 is involved in the regulation of phosphorylation of ERK or STAT3 in different cell contexts [42-46]. Secondly, although structurally similar, the role of SHP1 and SHP-2 in signaling is not redundant $[40,47]$. Thirdly, the phosphatase activity of SHP-1 and SHP-2 is dependent on the C-terminal phosphorylation at different tyrosine or serine residues [44, 48-51]. On the contrary, while the importance of the SHP-1 and SHP-2, as well as the ERK and STAT3 signaling in NK cell function are well known, it is still unclear whether SHP-1 or SHP-2 can directly dephosphorylate ERK and STAT3. Concerning the relationship between phosphatases and NK function, controversial studies have suggested that the expression of SHP-1 or SHP-2 may play opposite roles in regulate NK cell. For example, SHP-1 was demonstrated to be a major mediator of the inhibitory function of MHC class Ispecific receptors, and involved in promoting NK cell responsiveness against tumor [25]. In contrast, SHP2 was suggested to regulate NK cell function negatively [52]. More recently, SHP-2 was found to be a key mediator of IL-15-induced ERK activation, and is important for IL-15 stimulation-induced raise in glycolytic and respiration rates [53]. Herein, we demonstrated that hypoxia could activate both SHP-1 and SHP-2 by inducing phosphorylation, suggesting a possibility that SHP-1 and SHP-2 dephosphorylate ERK and STAT3. However, using specific blocking inhibitors of SHP-1 and SHP-2, we observed that targeting SHP-1 with TPI-1 alone could increase the phosphorylation of ERK and STAT3, and enhance NK cell cytotoxicity. However, SHP-2 inhibition had no effect on the phosphorylation level of ERK and STAT3. Furthermore, by silencing the gene expression of SHP-1 in KHYG-1 cells, we further confirmed the dephosphorylation effect on ERK and STAT3. Collectively, our findings show that hypoxia could impair NK cell cytotoxicity through decreasing the phosphorylation of ERK and STAT3 in a SHP-1 dependent manner.

Numerous studies have demonstrated that hypoxia could affect a large number of genes in many aspects of cells including proliferation, apoptosis, migration and metabolism, in multiple cell types in a hypoxia-inducible factors (HIFs) dependent manner. In this process, HIF1a and HIF2a are the central transcriptional regulators of the cellular response to low oxygen [54]. Although little is known about the effect of hypoxia on NK cells, a recent study based on comprehensive transcriptome analysis of hypoxic NK cells showed significant changes in genes coding for proinflammatory cytokines, chemokines, and chemokine-receptors [21]. Along these lines, it may suggest more complex mechanisms in hypoxiamediated NK cell dysfunction against tumors. Therefore, further study is still needed to clarify additional details as it relates to NK cell response to hypoxia, for instance, the molecular mechanisms of how hypoxia directly affects the phosphorylation of SHP-1, whether HIF1a or HIF2a transcriptionally regulates SHP-1.

In conclusion, we demonstrated that SHP-1 plays an important role in hypoxia-impaired NK cell cytotoxicity. Our finding supports that targeting SHP-1 may provide an important approach for improving NK cell-based tumor immunotherapy.

\section{Abbreviations}


DMEM - Dulbecco's modified Eagle's medium

ERK - Extracellular signal-regulated kinase

$\mathrm{E}: \mathrm{T}$ - Effector to target

FITC - Fluorescein isothiocyanate

ITAM - Intracytoplasmic tyrosine-based activation motifs

ITIM - Intracytoplasmic tyrosine-based inhibitory motifs

KAR - Killer activation receptors

KIR - Killer cell immunoglobulin-like receptors

MHC - Major histocompatibility complex

NK - Natural killer

PTK - Protein tyrosine kinase

PTP - Protein tyrosine phosphatase

SHP-1 - Src homology region 2 domain-containing phosphatase-1

SHP-2 - Src homology region 2 domain-containing phosphatase-2

STAT3 - Signal transducer and activator of transcription 3

\section{Declarations}

Acknowledgements The authors thank Prof. Baojun Zhang for help with flow cytometry analysis.

Funding This research was supported by grants from the National Natural Science Foundation of China, No. 81570192 and 81372534, and supported by an Openning Project of Key Laboratory of Shaanxi Province for Craniofacial Precision Medicine Research, College of Stomatology, Xi'an Jiaotong University (2018LHM-KFKT008).

Author contributions Conceived and designed the experiments: JH, MOD; Performed the experiments: YW, RT, NL, RA, LL, RF, YZ, PC, YP, BW, JF, XL; Analyzed the data: BW, JH, AH; Contributed reagents/materials/analysis tools: $\mathrm{MOD}, \mathrm{AH}$; Wrote the paper: $\mathrm{JH}, \mathrm{MOD}$. 
Conflict of interest MOD is Founder and Director of Onkimmune. The other authors have no potential conflicts of interest.

\section{References}

1. Guillerey C, Huntington ND, Smyth MJ (2016) Targeting natural killer cells in cancer immunotherapy. Nat Immunol 17:1025-1036

2. Sheard MA, Asgharzadeh S, Liu Y, Lin TY, Wu HW, Ji L, Groshen S, Lee DA, Seeger RC (2013) Membrane-bound TRAIL supplements natural killer cell cytotoxicity against neuroblastoma cells. J Immunother 36:319-329

3. Fauriat C, Long EO, Ljunggren HG, Bryceson YT (2010) Regulation of human NK-cell cytokine and chemokine production by target cell recognition. Blood 115:2167-2176

4. Davis ZB, Vallera DA, Miller JS, Felices M (2017) Natural killer cells unleashed: Checkpoint receptor blockade and BiKE/TriKE utilization in NK-mediated anti-tumor immunotherapy. Semin Immunol 31:64-75

5. Paul S, Lal G (2017) The Molecular Mechanism of Natural Killer Cells Function and Its Importance in Cancer Immunotherapy. Front Immunol 8:1124

6. Pegram HJ, Andrews DM, Smyth MJ, Darcy PK, Kershaw MH (2011) Activating and inhibitory receptors of natural killer cells. Immunol Cell Biol 89:216-224

7. Koch J, Steinle A, Watzl C, Mandelboim O (2013) Activating natural cytotoxicity receptors of natural killer cells in cancer and infection. Trends Immunol 34:182-191

8. Solomon BL, Garrido-Laguna I (2018) TIGIT: a novel immunotherapy target moving from bench to bedside. Cancer Immunol Immunother 67:1659-1667

9. Jandus C, Boligan KF, Chijioke O, Liu H, Dahlhaus M, Démoulins T, Schneider C, Wehrli M, Hunger RE, Baerlocher GM, Simon HU, Romero P, Münz C, von Gunten S (2014) Interactions between Siglec7/9 receptors and ligands influence NK cell-dependent tumor immunosurveillance. J Clin Invest 124:1810-1820

10. Roman Aguilera A, Lutzky VP, Mittal D, Li XY, Stannard K, Takeda K, Bernhardt G, Teng MWL, Dougall WC, Smyth MJ (2018) CD96 targeted antibodies need not block CD96-CD155 interactions to promote NK cell anti-metastatic activity. Oncoimmunology 7:e1424677

11. Lee H, Quek C, Silva I, Tasker A, Batten M, Rizos H, Lim SY, Nur Gide T, Shang P, Attrill GH, Madore J, Edwards J, Carlino MS, Guminski A, Saw RPM, Thompson JF, Ferguson PM, Palendira U, Menzies AM, Long GV, Scolyer RA, Wilmott JS (2018) Integrated molecular and immunophenotypic analysis of NK cells in anti-PD-1 treated metastatic melanoma patients. Oncoimmunology 8:e1537581

12. Hofer E, KoehI U (2017) Natural Killer Cell-Based Cancer Immunotherapies: From Immune Evasion to Promising Targeted Cellular Therapies. Front Immunol 8:745

13. Beatty GL, Gladney WL (2015) Immune escape mechanisms as a guide for cancer immunotherapy. Clin Cancer Res 21:687-692 
14. Petitprez F, Vano YA, Becht E, Giraldo NA, de Reynies A, Sautes-Fridman C, Fridman WH (2018) Transcriptomic analysis of the tumor microenvironment to guide prognosis and immunotherapies. Cancer Immunol Immunother 67:981-988

15. Yeh JJ, Kim WY (2015) Targeting tumor hypoxia with hypoxia-activated prodrugs. J Clin Oncol 33:1505-1508

16. Hu J, Van Valckenborgh E, Menu E, De Bruyne E, Vanderkerken K (2012) Understanding the hypoxic niche of multiple myeloma: therapeutic implications and contributions of mouse models. Dis Model Mech 5:763-771

17. Benito J, Ramirez MS, Millward NZ, Velez J, Harutyunyan KG, Lu H, Shi YX, Matre P, Jacamo R, Ma H, Konoplev S, McQueen T, Volgin A, Protopopova M, Mu H, Lee J, Bhattacharya PK, Marszalek JR, Davis RE, Bankson JA, Cortes JE, Hart CP, Andreeff M, Konopleva M (2016) Hypoxia-Activated Prodrug TH-302 Targets Hypoxic Bone Marrow Niches in Preclinical Leukemia Models. Clin Cancer Res 22:1687-1698

18. Azab AK, Hu J, Quang P, Azab F, Pitsillides C, Awwad R, Thompson B, Maiso P, Sun JD, Hart CP, Roccaro AM, Sacco A, Ngo HT, Lin CP, Kung AL, Carrasco RD, Vanderkerken K, Ghobrial IM (2012) Hypoxia promotes dissemination of multiple myeloma through acquisition of epithelial to mesenchymal transition-like features Blood 119:5782-5794

19. Hu J, Handisides DR, Van Valckenborgh E, De Raeve H, Menu E, Vande Broek I, Liu Q, Sun JD, Van Camp B, Hart CP, Vanderkerken K (2010) Targeting the multiple myeloma hypoxic niche with TH-302, a hypoxia-activated prodrug. Blood 116:1524-1527

20. Hasmim M, Messai Y, Ziani L, Thiery J, Bouhris JH, Noman MZ, Chouaib S (2015) Critical Role of Tumor Microenvironment in Shaping NK Cell Functions: Implication of Hypoxic Stress. Front Immunol 6:482

21. Parodi M, Raggi F, Cangelosi D, Manzini C, Balsamo M, Blengio F, Eva A, Varesio L, Pietra G, Moretta L, Mingari MC, Vitale M, Bosco MC (2018) Hypoxia Modifies the Transcriptome of Human NK Cells, Modulates Their Immunoregulatory Profile, and Influences NK Cell Subset Migration. Front Immunol $9: 2358$

22. Cacalano NA (2016) Regulation of Natural Killer Cell Function by STAT3. Front Immunol 7:128

23. Jiang K, Zhong B, Gilvary DL, Corliss BC, Hong-Geller E, Wei S, Djeu JY (2000) Pivotal role of phosphoinositide-3 kinase in regulation of cytotoxicity in natural killer cells. Nat Immunol 1(5):419425

24. Vivier E, Nunes JA, Vely F (2004) Natural killer cell signaling pathways. Science 306:1517-1519

25. Viant C, Fenis A, Chicanne G, Payrastre B, Ugolini S, Vivier E (2014) SHP-1-mediated inhibitory signals promote responsiveness and anti-tumour functions of natural killer cells. Nat Commun 5:5108

26. Barsoum IB, Koti M, Siemens DR, Graham CH (2014) Mechanisms of hypoxia-mediated immune escape in cancer. Cancer Res 74:7185-7190

27. Velasquez SY, Killian D, Schulte J, Sticht C, Thiel M, Lindner HA (2016) Short Term Hypoxia Synergizes with Interleukin 15 Priming in Driving Glycolytic Gene Transcription and Supports Human 
Natural Killer Cell Activities. J Biol Chem 291(25):12960-12977

28. Chambers AM, Matosevic S (2019) Immunometabolic Dysfunction of Natural Killer Cells Mediated by the Hypoxia-CD73 Axis in Solid Tumors. Front Mol Biosci 6:60

29. Sarkar S, Germeraad WT, Rouschop KM, Steeghs EM, van Gelder M, Bos GM, Wieten L (2013) Hypoxia induced impairment of NK cell cytotoxicity against multiple myeloma can be overcome by IL-2 activation of the NK cells. Plos One 8: e64835

30. Balsamo M, Manzini C, Pietra G, Raggi F, Blengio F, Mingari MC, Varesio L, Moretta L, Bosco MC, Vitale M (2013) Hypoxia downregulates the expression of activating receptors involved in NKcell-mediated target cell killing without affecting ADCC. Eur J Immunol 43:2756-2764

31. Wolter F, Glassner A, Kramer B, Kokordelis P, Finnemann C, Kaczmarek DJ, Goeser F, Lutz P, Nischalke HD, Strassburg CP, Spengler U, Nattermann J (2015) Hypoxia impairs anti-viral activity of natural killer (NK) cells but has little effect on anti-fibrotic NK cell functions in hepatitis $\mathrm{C}$ virus infection. J Hepatol 63: 1334-1344

32. Long EO, Kim HS, Liu D, Peterson ME, Rajagopalan S (2013) Controlling natural killer cell responses: integration of signals for activation and inhibition. Annu Rev Immunol 31:227-258

33. Kumar S (2018) Natural killer cell cytotoxicity and its regulation by inhibitory receptors. Immunology 154:383-393

34. Wagner JA, Rosario M, Romee R, Berrien-Elliott MM, Schneider SE, Leong JW, Sullivan RP, Jewell BA, Becker-Hapak M, Schappe T, Abdel-Latif S, Ireland AR, Jaishankar D, King JA, Vij R, Clement D, Goodridge J, Malmberg KJ, Wong HC, Fehniger TA (2017) CD56 bright NK cells exhibit potent antitumor responses following IL-15 priming. J Clin Invest 127:4042-4058

35. James AM, Hsu HT, Dongre P, Uzel G, Mace EM, Banerjee PP, Orange JS (2013) Rapid activation receptor- or IL-2-induced lytic granule convergence in human natural killer cells requires Src, but not downstream signaling. Blood 121:2627-2637

36. Suck G, Branch DR, Aravena P, Mathieson M, Helke S, Keating A (2006) Constitutively polarized granules prime KHYG-1 NK cells. Int Immunol 18:1347-1354

37. Abeyweera TP, Merino E, Huse M (2011) Inhibitory signaling blocks activating receptor clustering and induces cytoskeletal retraction in natural killer cells. J Cell Biol 192:675-690

38. Yusa S, Catina TL, Campbell KS (2004) KIR2DL5 can inhibit human NK cell activation via recruitment of Src homology region 2-containing protein tyrosine phosphatase-2 (SHP-2). J Immunol 172:73857392.

39. Viant C, Fenis A, Chicanne G, Payrastre B, Ugolini S, Vivier E (2014) SHP-1-mediated inhibitory signals promote responsiveness and anti-tumour functions of natural killer cells. Nat Commun 5:5108

40. Pao LI, Badour K, Siminovitch KA, Neel BG (2007) Nonreceptor protein-tyrosine phosphatases in immune cell signaling. Annu Rev Immunol 25:473-523

41. Mazharian A, Mori J, Wang YJ, Heising S, Neel BG, Watson SP, Senis YA (2013) Megakaryocytespecific deletion of the protein-tyrosine phosphatases Shp1 and Shp2 causes abnormal megakaryocyte development, platelet production, and function. Blood 121:4205-4220 
42. Jia WQ, Wang ZT, Zou MM, Lin JH, Li YH, Zhang L, Xu RX (2018) Verbascoside Inhibits Glioblastoma Cell Proliferation, Migration and Invasion While Promoting Apoptosis Through Upregulation of Protein Tyrosine Phosphatase SHP-1 and Inhibition of STAT3 Phosphorylation. Cell Physiol Biochem 47:1871-1882

43. Wang Q, Downey GP, Herrera-Abreu MT, Kapus A, McCulloch CA (2005) SHP-2 modulates interleukin1-induced $\mathrm{Ca} 2+$ flux and ERK activation via phosphorylation of phospholipase Cgamma1. J Biol Chem 280:8397-8406

44. Wang Q, Herrera AM, Siminovitch K, Downey GP, McCulloch CA (2006) Phosphorylation of SHP-2 regulates interactions between the endoplasmic reticulum and focal adhesions to restrict interleukin1-induced Ca2+ signaling. J Biol Chem 281:31093-31105

45. Zhan Y, O'Rourke DM (2004) SHP-2-dependent mitogen-activated protein kinase activation regulates EGFRvIII but not wild-type epidermal growth factor receptor phosphorylation and glioblastoma cell survival. Cancer Res 64:8292-8298

46. Huang TT, Su JC, Liu CY, Shiau CW, Chen KF (2017) Alteration of SHP-1/p-STAT3 Signaling: A Potential Target for Anticancer Therapy. Int J Mol SCI 18

47. Wang N, Li Z, Ding R, Frank GD, Senbonmatsu T, Landon EJ, Inagami T, Zhao ZJ (2006) Antagonism or synergism. Role of tyrosine phosphatases SHP-1 and SHP-2 in growth factor signaling. J Biol Chem 281:21878-21883

48. Zhang Z, Shen K, Lu W, Cole PA (2003) The role of C-terminal tyrosine phosphorylation in the regulation of SHP-1 explored via expressed protein ligation. J Biol Chem 278:4668-4674

49. Lu W, Gong D, Bar-Sagi D, Cole PA (2001) Site-specific incorporation of a phosphotyrosine mimetic reveals a role for tyrosine phosphorylation of SHP-2 in cell signaling. Mol Cell 8:759-769.

50. Jones ML, Craik JD, Gibbins JM, Poole AW (2004) Regulation of SHP-1 tyrosine phosphatase in human platelets by serine phosphorylation at its $C$ terminus. J Biol Chem 279:40475-40483

51. Liu Y, Kruhlak MJ, Hao JJ, Shaw S (2007) Rapid T cell receptor-mediated SHP-1 S591 phosphorylation regulates SHP-1 cellular localization and phosphatase activity. J Leukoc Biol 82:742-751

52. Purdy AK, Campbell KS (2009) SHP-2 expression negatively regulates NK cell function. J Immunol 183(11):7234-7243

53. Niogret C, Miah S, Rota G, Fonta NP, Wang H, Held W, Birchmeier W, Sexl V, Yang W, Vivier E and others (2019) Shp-2 is critical for ERK and metabolic engagement downstream of IL-15 receptor in NK cells. Nat Commun 10(1):1444

54. Majmundar AJ, Wong WJ, Simon MC (2010) Hypoxia-inducible factors and the response to hypoxic stress. Mol Cell 40(2):294-309

\section{Figures}


a
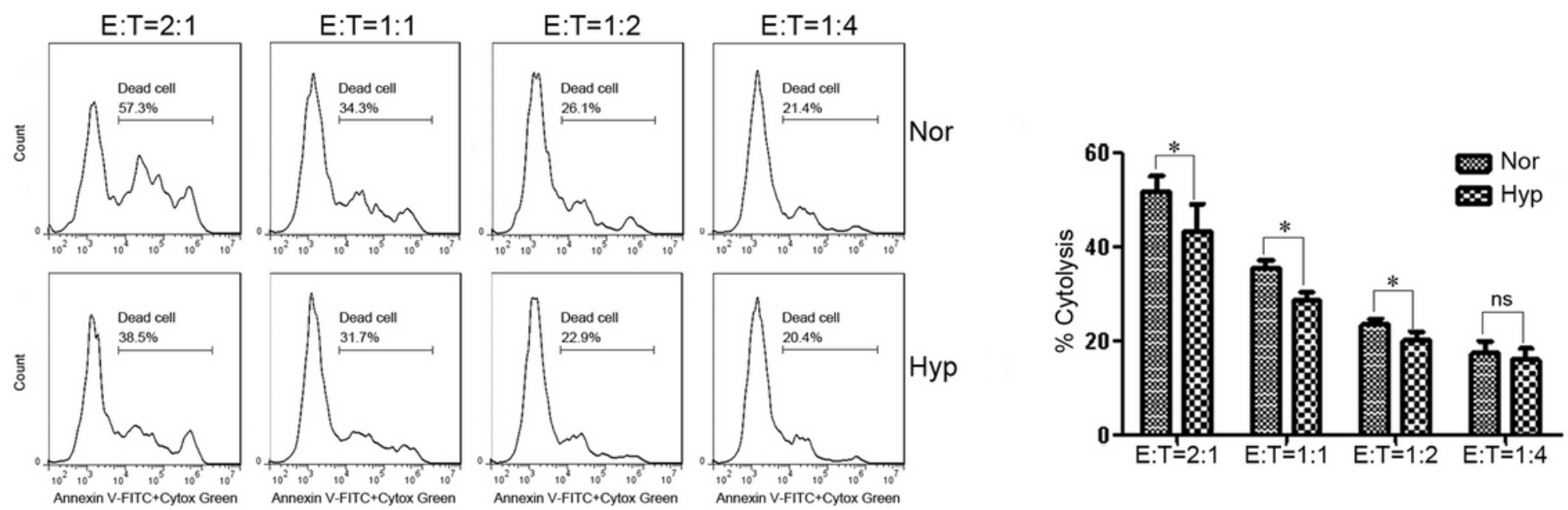

b
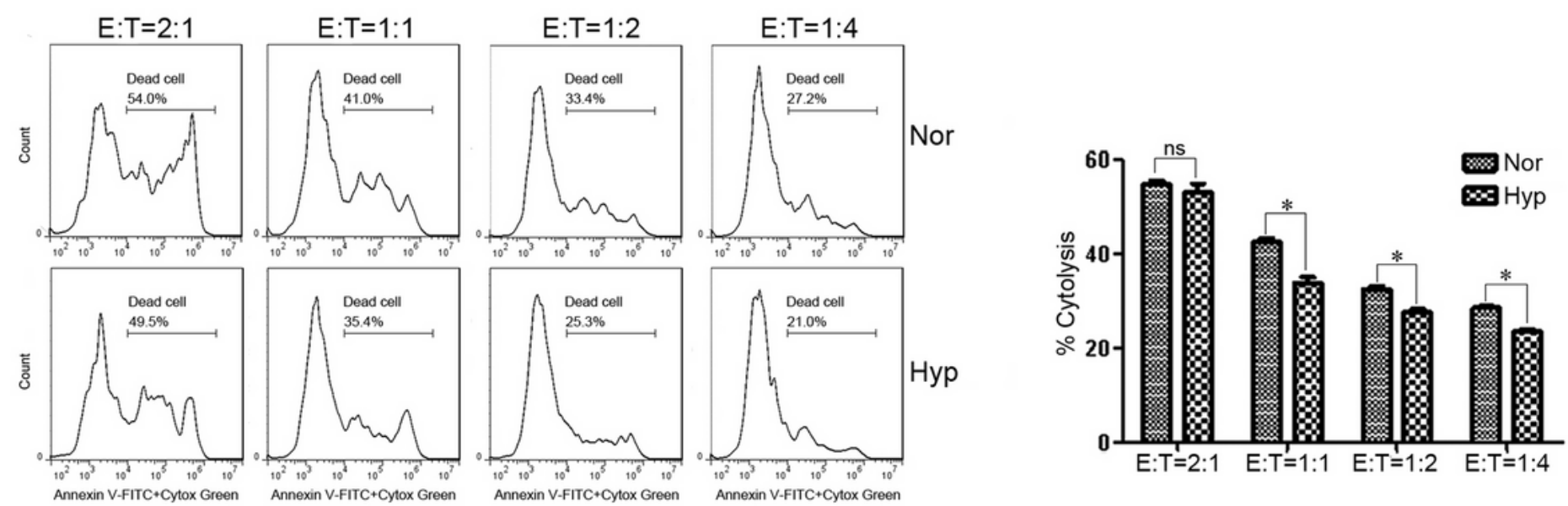

C

d

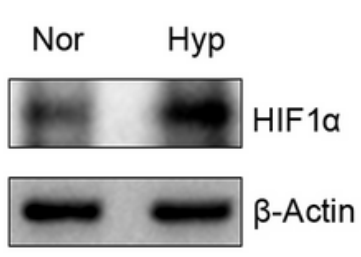

KHYG-1

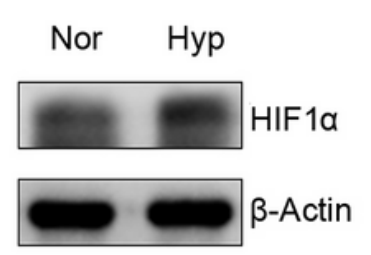

NK92

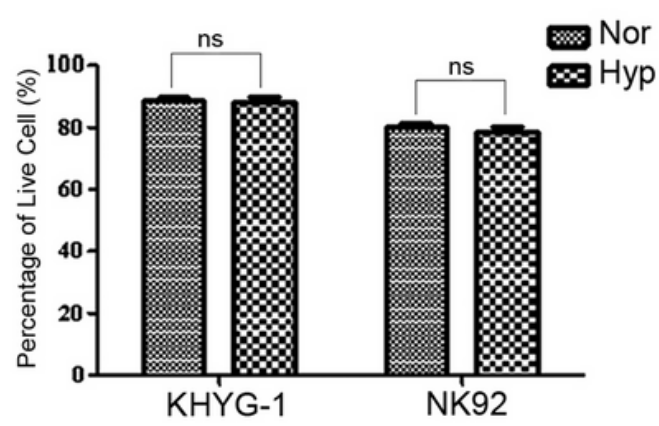

\section{Figure 1}

Hypoxic NK cells show lower cytotoxicity against tumor cells. (a-b) Flow cytometric analysis of KHYG-1 cells cytotoxicity against tumor cells. KHYG-1 cells were incubated with K562 (a) or MM.1S (b) tumor cells for $4 \mathrm{~h}$ at different E:T ratios after cultivation at normoxic ( $20 \% 02)$ and hypoxic ( $1 \% 02)$ conditions for $24 \mathrm{~h}$ (left panel, a representation of results from three experiments; right panel, statistical analysis showing the percentage of tumor cells killed by NK cells.) (c) Western blotting analysis of the effects of 
hypoxia on the expression of hypoxia marker HIF-1a. NK cells were cultured in $20 \%$ or $1 \% 02$ for $24 \mathrm{~h}$, then western blotting analysis was performed. (d) Flow cytometric analysis of the effects of hypoxia on NK cell viability by performing Annexin V-FITC/7-AAD staining $(n=3, * P<0.05, * * P<0.01)$. NK cells were cultured in $20 \%$ or $1 \% 02$ for $24 \mathrm{~h}$, then flow cytometric staining was performed.

a Granzyme B
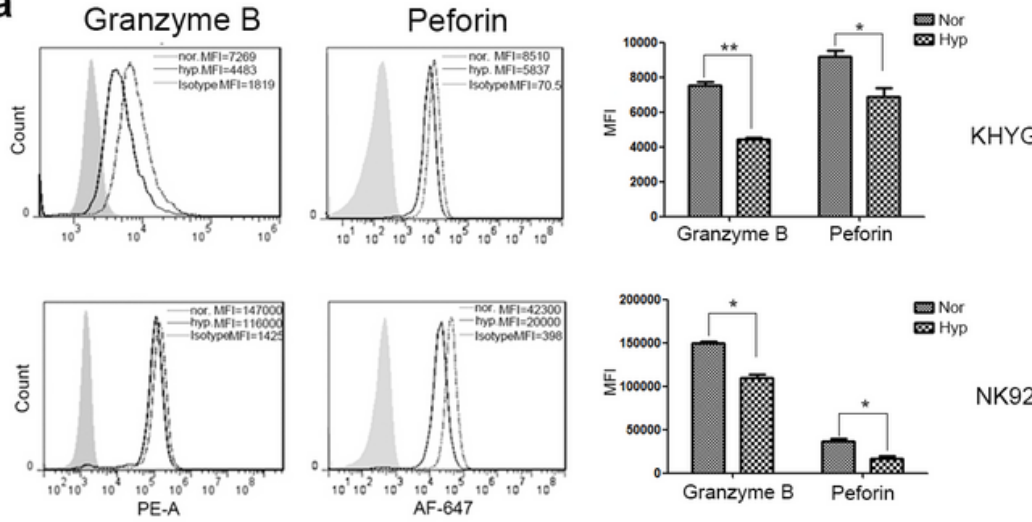

d
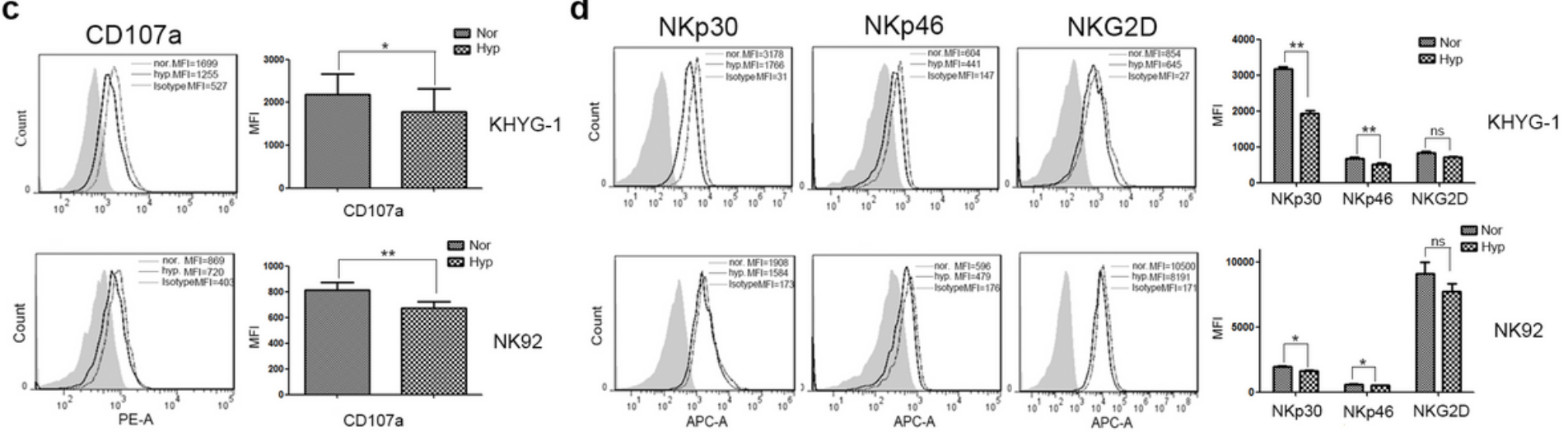

Figure 2

Hypoxia decreases the expression level of NK cells cytotoxicity related molecules. (a) Flow cytometric analysis of granzyme and perforin expression in KHYG-1 (upper panel) and NK92 (lower panel) cells, respectively. KHYG-1 and NK92 were cultured in normoxic (20\% 02) and hypoxic ( $1 \% 02)$ for $24 \mathrm{~h}$, then intracellular staining was performed to analyze the expression of granzyme and perforin quantitatively. Left panel: one of three representative flow cytometry results; Right panel: statistical analysis of the flow cytometry data $(n=3, * P<0.05$, $* * P<0.01)$. (b) Flow cytometric analysis of the intracellular level of IFN- $y$ in normoxic and hypoxic KHYG-1 and NK92 cells. Left panel: one of three representative flow cytometry results; Right panel: statistical analysis of the flow cytometry data $(n=3, * P<0.05, * \star P<0.01)$. (c) Flow cytometric analysis of the membrane staining of degranulation marker CD107a in normoxic and hypoxic KHYG-1 and NK92 cells. Left panel: one of three representative flow cytometry results; Right panel: statistical analysis of the flow cytometry data $(n=3, * P<0.05$, ** $P<0.01)$. (d) Flow cytometric analysis showing the membrane staining of activating receptor NKp30, NKp46, and NKG2D in normoxic and hypoxic KHYG-1 and NK92 cells. Representative data of three individual experiments. Left panel: one of three representative flow cytometry results; Right panel: statistical analysis of the flow cytometry data $(n=3$, * $P<0.05$, ** $P<0.01)$. 
a

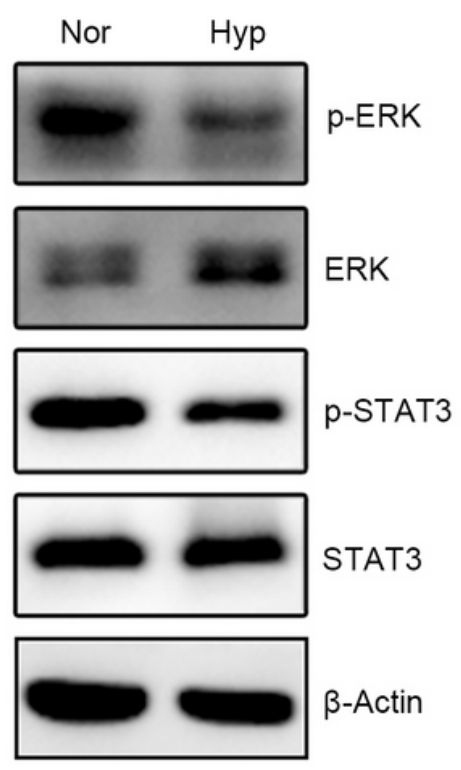

KHYG-1

b

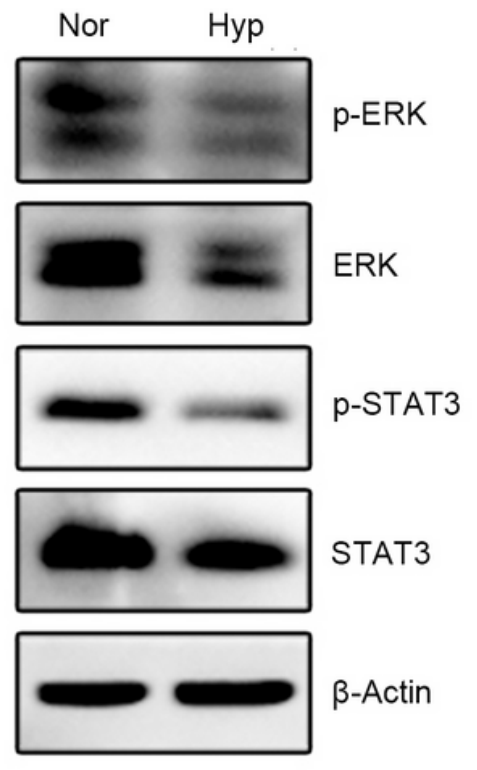

NK92

C
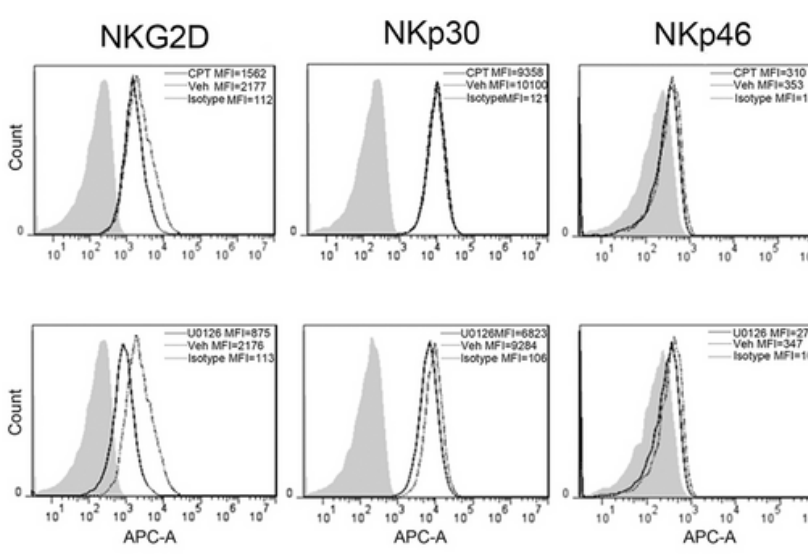

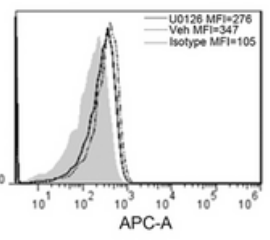

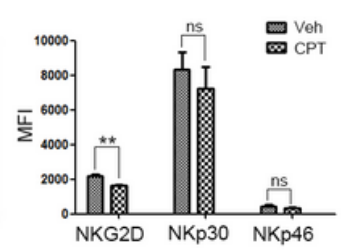

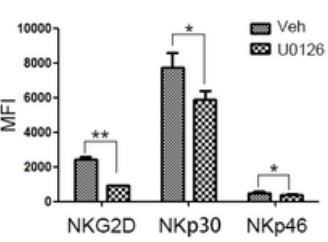

d

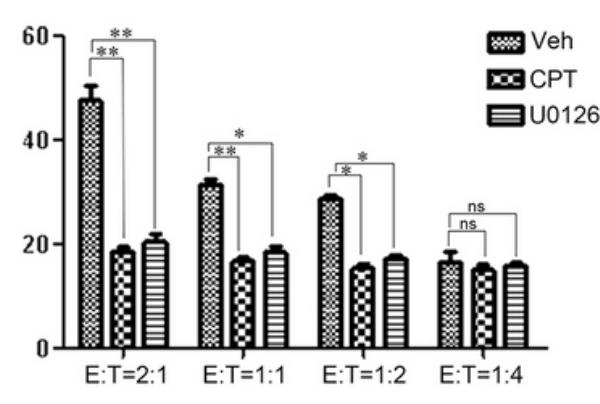

\section{Figure 3}

Hypoxia diminishes the phosphorylation level of ERK and STAT3. (a-b) Western blotting analysis showing the expression level of the phosphorylated ERK and STAT3 in KHYG-1 (a) and NK92 (b) cells, respectively. (c) Inhibition of ERK and STAT3 decreases the expression of activating receptors on the NK cell surface. Representative flow cytometry results of STAT3 inhibitor cryptotanshinone (CPT) (upper panel) and ERK inhibitor U0126 (lower panel) on the expression of activating receptors on NK cell surface. Left panel: one of three representative flow cytometry results; Right panel: statistical analysis of the flow cytometry data $(n=3, * P<0.05$, ** $P<0.01)$. KHYG-1 and NK92 cells were treated with vehicle, $10 \mu M$ ERK inhibitor U0126, and $10 \mu \mathrm{M}$ STAT3 inhibitor CPT for $24 \mathrm{~h}$. (d) Inhibition of ERK and STAT3 decreases NK cell cytotoxicity. Statistical analysis showing the effects of ERK and STAT3 inhibition on NK cells cytotoxicity against K562 cells $(n=3, * P<0.05$, ** P<0.01). KHYG-1 cells were pretreated with $10 \mu \mathrm{M}$ U0126 and $10 \mu \mathrm{M}$ CPT for $6 \mathrm{~h}$, then incubated with $\mathrm{K} 562$ at different $\mathrm{E}: \mathrm{T}$ ratios for $4 \mathrm{~h}$. 
a

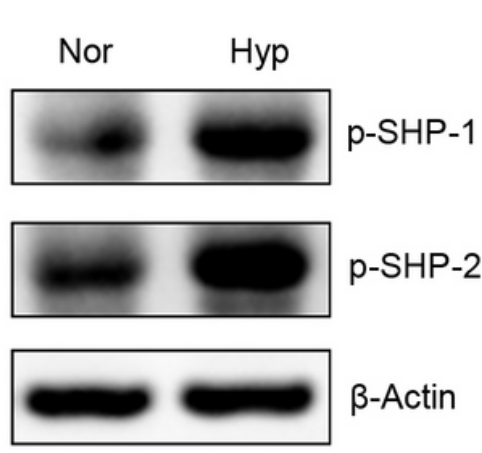

KHYG-1 b

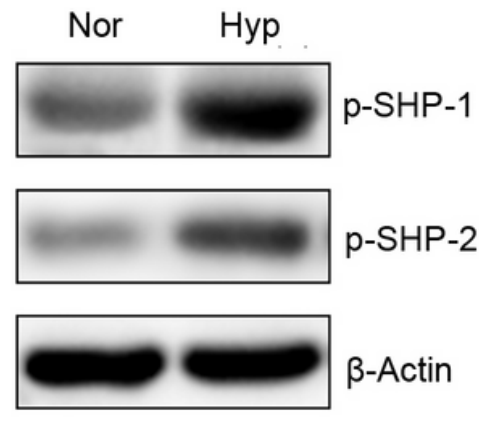

NK92
C
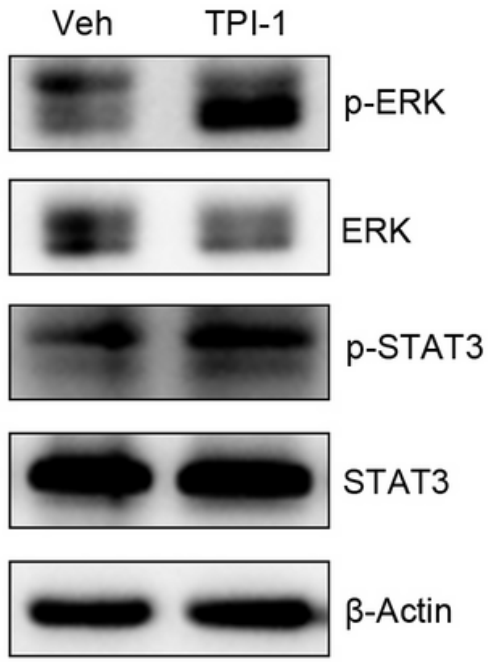

STAT3

$\beta$-Actin d
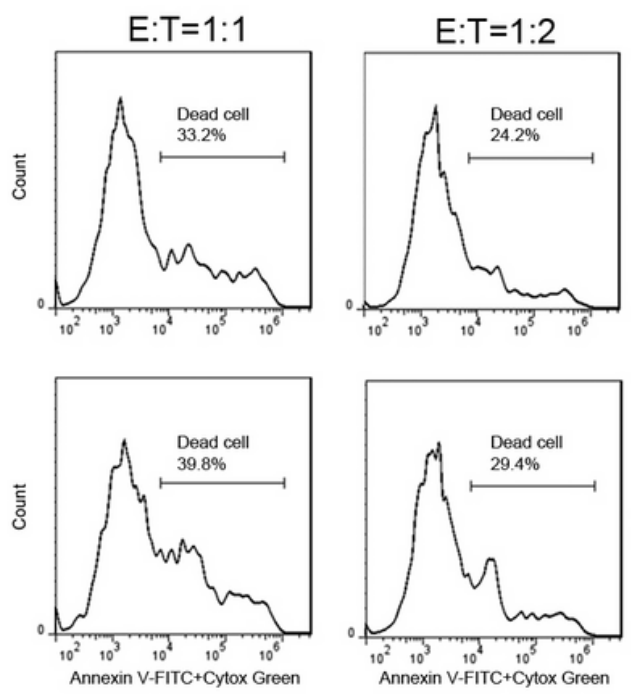
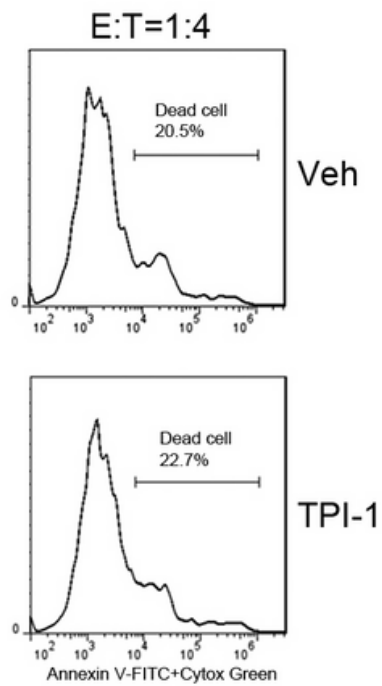

KHYG-1

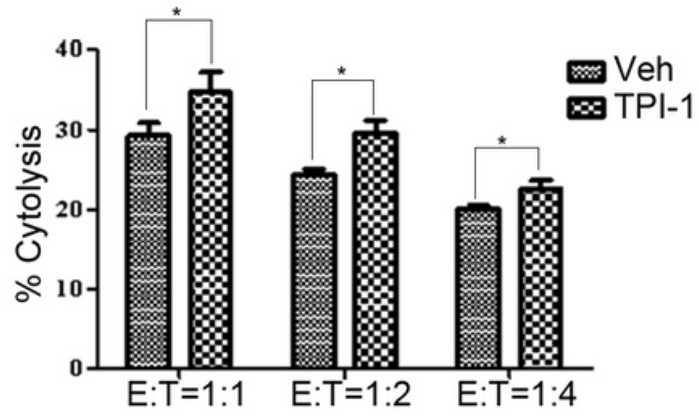

Figure 4

Hypoxia activates SHP-1 and SHP-2 in NK cells. (a-b) Western blotting analysis of p-SHP-1 and p-SHP-2 expression in normoxic ( $20 \%$ 02) and hypoxic (1\% 02) KHYG-1 (a) and NK92 (b) cells, respectively. (c) Western blotting showing the effects of SHP-1 inhibitor TPI-1 on the phosphorylation of ERK and STAT3. Hypoxic KYHG-1 cells were pre-treated with $5 \mu \mathrm{M}$ TPI- 1 for $2 \mathrm{~h}$, then the cells were collected for western blotting analysis. (d) Flow cytometric analysis of the effects of TPI-1 on the NK cell cytotoxicity. Left panel: representative flow cytometry results of TPI- 1 on the cytotoxicity of KHYG-1 cells. KHYG-1 cells were pretreated with $5 \mu \mathrm{M} \mathrm{TPI}-1$ for $2 \mathrm{~h}$, then incubated with K562 cells at different E:T ratios for $4 \mathrm{~h}$. Right panel: statistical analysis of the effects of TPI- 1 on KHYG-1 cell cytotoxicity against K562 cells ( $n=3, * P<0.05)$. 
a

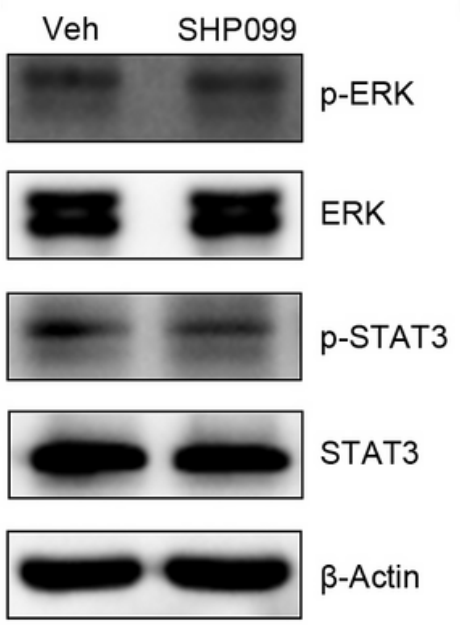

b
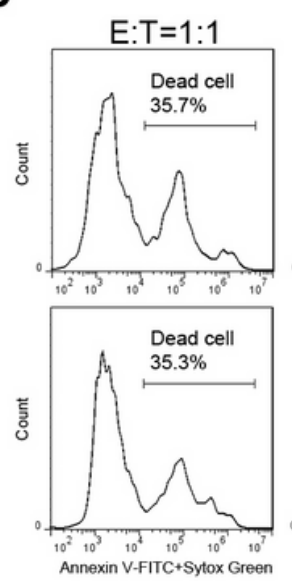

$E: T=1: 2$
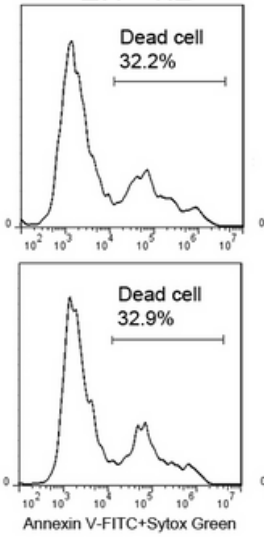

$\mathrm{E}: \mathrm{T}=1: 4$
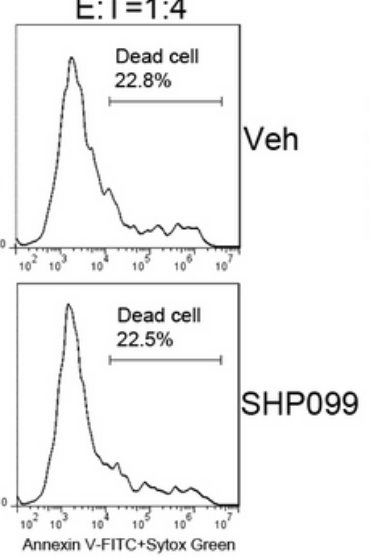

C

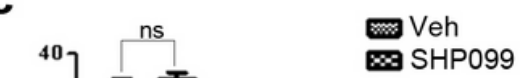

KHYG-1

\section{Figure 5}

Inhibition of SHP-2 has no effect on NK cell cytotoxicity. (a) Western blotting showing the effects of SHP2 inhibitor SHP099 on the phosphorylation of ERK and STAT3. Hypoxic KYHG-1 cells were pre-treated with $5 \mu \mathrm{M}$ SHP099 for $2 \mathrm{~h}$, then the cells were collected for western blotting analysis. (b) Flow cytometric analysis showing the effect of SHP099 on the cytotoxicity of KHYG-1 cells. KHYG-1 cells were pretreated with $5 \mu \mathrm{M}$ SHP099 for $2 \mathrm{~h}$, then incubated with K562 cells at different E:T ratios for $4 \mathrm{~h}$. (c) Statistical analysis showing the effects of SHP099 on KHYG-1 cell cytotoxicity against K562 cells $(n=3)$. 
a

si-control si-SHP-1

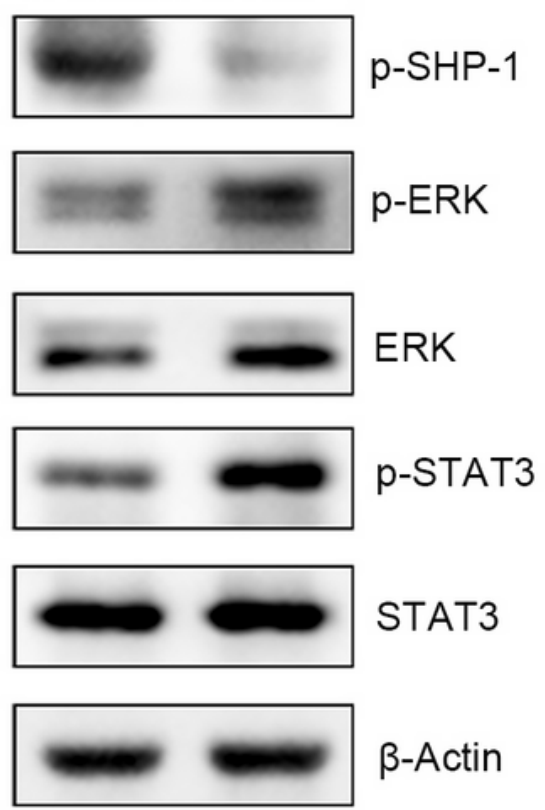

KHYG-1

\section{b}

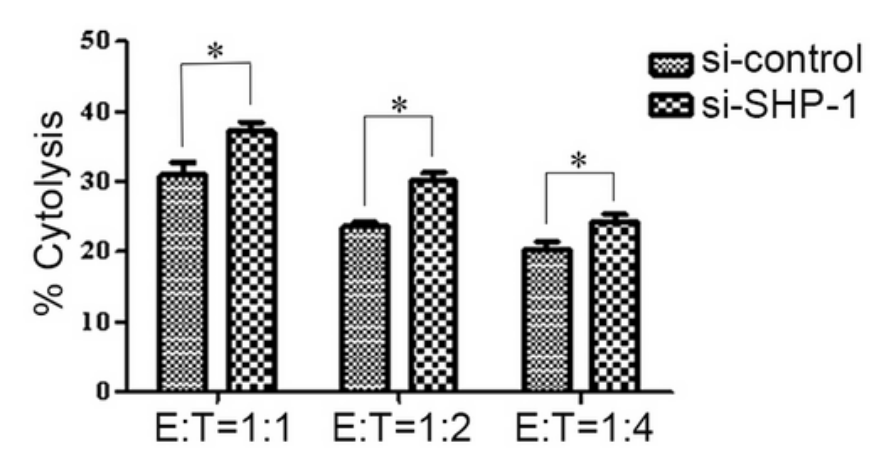


electroporated cells were used for western blotting or killing assay as previously mentioned. (c) A schematic diagram shows how hypoxia impairs NK cell cytotoxicity in a SHP-1 dependent manner.

\section{Supplementary Files}

This is a list of supplementary files associated with this preprint. Click to download.

- SupplementaryTable1.docx

- supplementalfugure1.tif 